\title{
On the Inverse Eigenvalue Problem for Irreducible Doubly Stochastic Matrices of Small Orders
}

\author{
Quanbing Zhang, ${ }^{1}$ Changqing $X u^{2}$ and Shangjun Yang ${ }^{3}$ \\ ${ }^{1}$ Key Laboratory of Intelligent Computing \& Signal Processing, Ministry of Education, Anhui University, Hefei, \\ Anhui 230039, China \\ ${ }^{2}$ Department of Applied Mathematics, Suzhou University of Science and Technology, Suzhou, Jiangsu 215009, China \\ ${ }^{3}$ School of Mathematical Sciences, Anhui University, Hefei, Anhui 230039, China
}

Correspondence should be addressed to Changqing Xu; cqxurichard@gmail.com

Received 28 July 2013; Accepted 17 January 2014; Published 4 March 2014

Academic Editor: Shusen Ding

Copyright ( 2014 Quanbing Zhang et al. This is an open access article distributed under the Creative Commons Attribution License, which permits unrestricted use, distribution, and reproduction in any medium, provided the original work is properly cited.

\begin{abstract}
The inverse eigenvalue problem is a classical and difficult problem in matrix theory. In the case of real spectrum, we first present some sufficient conditions of a real $r$-tuple (for $r=2 ; 3 ; 4 ; 5$ ) to be realized by a symmetric stochastic matrix. Part of these conditions is also extended to the complex case in the case of complex spectrum where the realization matrix may not necessarily be symmetry. The main approach throughout the paper in our discussion is the specific construction of realization matrices and the recursion when the targeted $r$-tuple is updated to a $(r+1)$-tuple.
\end{abstract}

\section{Introduction}

For a square matrix $A$, let $\sigma(A)$ denote the spectrum of $A$. Given an $n$-tuple $\Lambda=\left(\lambda_{1}, \ldots, \lambda_{n}\right)$ of numbers, real or complex, the problem of deciding the existence of a nonnegative matrix $A$ with $\sigma(A)=\Lambda$ is called the nonnegative inverse eigenvalue problem (NIEP) which has for a long time been one of the problems of main interest in the theory of matrices.

Sufficient conditions for the existence of an entrywise positive matrix $A$ with $\sigma(A)=\Lambda$ have been investigated by many authors [1-14]. The case $n=2$ is trivial. The problem has been solved for $n=3$ by Loewy and London [6]. The cases $n=4$ and $n=5$ have been solved for matrices with trace zero by Reams [10] and Laffey and Meehan [5], respectively. So, for real spectra, complete constructive solutions to NIEP are available for $n \leq 4$. For the case of nonreal spectra for $n=4$, complete solutions are available through the work of Laffey and Meehan [5], independently, and that of Torre-Mayo et al. [12] by analyzing coefficients of the characteristic polynomial. EBL digraphs, and for $n=2,3,4,5$, complete solutions are available through the work of Nazari and Sherafat [8].

An $n \times n$ nonnegative matrix $A=\left(a_{i j}\right)$ is called a row stochastic matrix if $\sum_{j=1}^{n} a_{i j}=1, i=1, \ldots, n ; A$ is called a doubly stochastic matrix if $\sum_{j=1}^{n} a_{i j}=1, i=1, \ldots, n$, and $\sum_{i=1}^{n} a_{i j}=1, j=1, \ldots, n$. Since row stochastic and doubly stochastic matrices are important nonnegative matrices, it is surely important to investigate the existence of row or doubly stochastic matrices with prescribed spectrum under certain conditions. We call this special NIEP the row or doubly stochastic inverse eigenvalue problem (RSIEP or DSIEP). Hwang and Pyo [3] gave some interesting results for the symmetric DSIEP.

An $n$-tuple $\Lambda=\left(\lambda_{1}, \ldots, \lambda_{n}\right)$ is nonnegative (doubly stochastic) realizable if there exists an $n \times n$ nonnegative (doubly stochastic) matrix $A$ with $\sigma(A)=\Lambda$. In this case, we say $A$ is a nonnegative (doubly stochastic) realization of $\Lambda$ or the nonnegative (doubly stochastic) matrix $A$ that realizes the $n$-tuple $\Lambda$.

A nonincreasing $n$-tuple $\Lambda=\left(\lambda_{1}, \ldots, \lambda_{n}\right)$ is called $S$ feasible if it satisfies

(1) $\sum_{j=1}^{n} \lambda_{j} \geq 0$;

(2) $1 \equiv \lambda_{1} \geq\left|\lambda_{j}\right|$ for all $j=2, \ldots, n$.

Throughout the paper, we denote the spectrum of $A$ by $\sigma(A)$; the spectrum radius of $A$ by $\rho(A)$; the all-ones column 
vector of $n$ dimensions by $e \in R^{n}$. We use $u_{n}$ for the $n$ dimensional normalized vector $(1 / \sqrt{n}) e^{T}$ and $I_{n}$ for the identity matrix of order $n$.

Theorem NN (see [15]). Let A be an irreducible nonnegative matrix of order $n$. Then, we have

(a) $\rho(A)>0$ and $\rho(A) \in \sigma(A)$;

(b) $A(x)=\rho(A) x$ for some $x$ in $\mathbf{R}^{\mathbf{n}}$, and the null space of $A-\rho(A) I_{n}$ is of dimension 1;

(c) $|\lambda|<\rho(A)$ for any $\lambda \neq \rho(A), \lambda \in \sigma(A)$.

In this paper, we study DSIEP of order $n \in\{2,3,4,5\}$. In Section 2, we present some sufficient conditions for the DSIEP for a given real $n$-tuple. In Section 3 , we present some sufficient conditions for the DSIEP for a given nonreal complex $n$-tuple where the realization matrix may not necessarily be symmetric.

\section{The Case of Real Spectrum}

Lemma 1. If $1>\lambda_{2} \geq-1$, then there is a $2 \times 2$ irreducible doubly stochastic matrix realizing $\Lambda=\left\{1, \lambda_{2}\right\}$.

Proof. It is easy to verify that the following $2 \times 2$ irreducible doubly stochastic matrix:

$$
\frac{1}{2}\left(\begin{array}{ll}
1+\lambda_{2} & 1-\lambda_{2} \\
1-\lambda_{2} & 1+\lambda_{2}
\end{array}\right)
$$

realizes $\Lambda=\left\{1, \lambda_{2}\right\}$.

In this section, we present a theorem that is analogy to Theorem 2.1 of [8]. The theorem is used to construct an $n \times n$ irreducible symmetric stochastic realization of a given $n$-tuple with designed conditions.

Theorem 2. For any integer $m \geq 2$ if $B$ is an $m \times m$ irreducible doubly stochastic matrix with $\sigma(B)=\left\{1, \mu_{2}, \ldots, \mu_{m}\right\}$ and $1>$ $\lambda \geq-1$, then there exists an $(m+1) \times(m+1)$ irreducible doubly stochastic matrix $C$ such that $\sigma(C)=\left\{1, c \mu_{2}, \ldots, c \mu_{m}, \mu_{m+1}\right\}$, where $c=1-(1-\lambda) / 2 m=(2 m-1+\lambda) / 2 m>0 ; \mu_{m+1}=$ $((m+1) \lambda+m-1) / 2 m$.

Proof. We know, by Theorem NN, that $\rho(B)=1$ is a simple eigenvalue of $B$ and $u_{m}$ is the unique normalized positive eigenvector associated with 1 such that $u_{m}^{T} u_{m}=1, B u_{m}=u_{m}$, $u_{m}^{T} B=u_{m}^{T}$. Now we can find an $m \times(m-1)$ matrix $V_{1}$ such that $Y_{1}=\left(u_{m}, V_{1}\right)$ is a unitary matrix. Then,

$$
B_{1}=Y_{1}^{*} B Y_{1}=\left(\begin{array}{ll}
u_{m}^{T} u_{m} & u_{m}^{T} B V_{1} \\
V_{1}^{*} u_{m} & V_{1}^{*} B V_{1}
\end{array}\right)=\left(\begin{array}{cc}
1 & * \\
0 & \widehat{B}
\end{array}\right),
$$

where $\left(V_{1}^{*} u_{m}\right)^{*}=u_{m}^{T} V_{1}=0, \widehat{B}=V_{1}^{*} B V_{1}$ and it is not necessary to know the value of each entry in the location remarked by " $*$." Since $\sigma\left(B_{1}\right)=\sigma(B)=\left\{1, \mu_{2}, \ldots, \mu_{m}\right\}$, we have $\sigma(\widehat{B})=\left\{\mu_{2}, \ldots, \mu_{m}\right\}$. By Schur's unitary triangularization theorem (see [15]), there exists a unitary matrix $V_{2}$ of order $m-1$, such that $V_{2}^{*} \widehat{B} V_{2}=\widehat{T}_{B}$, where $\widehat{T}_{B}$ is a upper triangular matrix and $\sigma(\widehat{B})$ is the set of all diagonal entries of $\widehat{T}_{B}$. Now for the $m \times m$ unitary matrix $Y_{2}=(1) \oplus V_{2}$, we have

$$
Y_{2}^{*} B_{1} Y_{2}=Y_{2}^{*}\left(Y_{1}^{*} B Y_{1}\right) Y_{2}=\left(Y_{1} Y_{2}\right)^{*} B\left(Y_{1} Y_{2}\right)=Y^{*} B Y \text {, }
$$

where $Y=Y_{1} Y_{2}=\left(u_{m}, V_{1} V_{2}\right)=\left(u_{m}, T\right)$ is unitary with $T=$ $V_{1} V_{2}$ and

$$
\begin{gathered}
Y Y^{*}=u_{m} u_{m}^{T}+T T^{*}=I_{m}, \\
Y^{*} Y=\left(\begin{array}{ll}
u_{m}^{T} u_{m} & u_{m}^{T} T \\
T^{*} u_{m} & T^{*} T
\end{array}\right)=(1) \oplus I_{m-1} .
\end{gathered}
$$

Therefore,

$$
\begin{gathered}
u_{m}^{T} T=0, \quad T^{*} u_{m}=0, \quad T^{*} T=I_{m-1}, \\
Y^{*} B Y=\left(\begin{array}{cc}
u_{m}^{T} B u_{m} & u_{m}^{T} B T \\
T^{*} B u_{m} & T^{*} B T
\end{array}\right)=\left(\begin{array}{cc}
1 & * \\
0 & T^{*} B T
\end{array}\right),
\end{gathered}
$$

where $T^{*} B u_{m}=T^{*} u_{m}=0$ by (5) and

$$
\sigma\left(T^{*} B T\right)=\sigma\left(Y^{*} B Y\right)-\{1\}=\sigma(B)-\{1\}=\left\{\mu_{2}, \ldots, \mu_{m}\right\} .
$$

It is easy to verify that the following $(m+1) \times(m+1)$ matrix:

$$
\begin{aligned}
C & =\left(\begin{array}{cc}
\frac{1+\lambda}{2} & \frac{1-\lambda}{2 \sqrt{m}} u_{m}^{T} \\
\frac{1-\lambda}{2 \sqrt{m}} u_{m} & \left(1-\frac{(1-\lambda) / 2}{m}\right) B
\end{array}\right) \\
& =\left(\begin{array}{cc}
\frac{1+\lambda}{2} & \frac{1-\lambda}{2 \sqrt{m}} u_{m}^{T} \\
\frac{1-\lambda}{2 \sqrt{m}} u_{m} & c B
\end{array}\right)
\end{aligned}
$$

is a doubly stochastic matrix. Let $\beta=(1 / \sqrt{2},-1 / \sqrt{2})^{T}$; then the following $(m+1) \times(m+1)$ matrix:

$$
Z=\left(\begin{array}{cc}
u_{2}^{T} & 0 \\
u_{m} \beta^{T} & T
\end{array}\right)
$$

is a unitary matrix, since

$$
Z Z^{*}=\left(\begin{array}{cc}
u_{2}^{T} u_{2} & 0 \\
0 & T T^{*}
\end{array}\right)=\left(\begin{array}{cc}
1 & 0 \\
0 & I_{m}
\end{array}\right)
$$

by $u_{2}^{T} \beta=0, \beta^{T} u_{2}=0$, and $u_{m} u_{m}^{T}+T T^{*}=I_{m}$. In addition using $u_{m}^{T} u_{m}=1$ and $T^{*} B u_{m}=T^{*} u_{m}=0$, we have

$Z^{*} C Z$

$$
\begin{aligned}
& =\left(\begin{array}{cc}
\frac{1+\lambda}{2} u_{2} u_{2}^{T}+\frac{1-\lambda}{2 \sqrt{m}} \beta u_{2}^{T}+\frac{1-\lambda}{2 \sqrt{m}} u_{2} \beta^{T}+c \beta \beta^{T} & 0 \\
0 & c T^{*} B T
\end{array}\right) \\
& =\left(\begin{array}{cc}
C_{1} & 0 \\
0 & c T^{*} B T
\end{array}\right),
\end{aligned}
$$


where

$$
\begin{aligned}
C_{1}= & \frac{1+\lambda}{2} u_{2} u_{2}^{T}+\frac{1-\lambda}{2 \sqrt{m}} \beta u_{2}^{T}+\frac{1-\lambda}{2 \sqrt{m}} u_{2} \beta^{T}+c \beta \beta^{T} \\
= & \left(\begin{array}{cc}
\frac{1+\lambda}{4} & \frac{1+\lambda}{4} \\
\frac{1+\lambda}{4} & \frac{1+\lambda}{4}
\end{array}\right)+\left(\begin{array}{cc}
\frac{1-\lambda}{8 \sqrt{m}} & \frac{1-\lambda}{8 \sqrt{m}} \\
-\frac{1-\lambda}{8 \sqrt{m}} & -\frac{1-\lambda}{8 \sqrt{m}}
\end{array}\right) \\
& +\left(\begin{array}{cc}
\frac{1-\lambda}{8 \sqrt{m}} & -\frac{1-\lambda}{8 \sqrt{m}} \\
\frac{1-\lambda}{8 \sqrt{m}} & -\frac{1-\lambda}{8 \sqrt{m}}
\end{array}\right)+\left(\begin{array}{cc}
\frac{c}{2} & -\frac{c}{2} \\
-\frac{c}{2} & \frac{c}{2}
\end{array}\right) .
\end{aligned}
$$

Therefore, $\sigma(C)=\sigma\left(C_{1}\right) \cup \sigma\left(c T^{*} B T\right)=\sigma\left(C_{1}\right) \cup\left\{c \mu_{2}, \ldots, c \mu_{m}\right\}$. Since $0<c<1$, we have $\left|c \mu_{i}\right|<1, i=2, \ldots, m$ and hence $1 \notin\left\{c \mu_{2}, \ldots, c \mu_{m}\right\}$. In addition, $1 \in \sigma(C)$ (for $C$ is a doubly stochastic matrix) implies that $1 \in \sigma\left(C_{1}\right)$. So the spectrum of $C_{1}$ is $\sigma\left(C_{1}\right)=\left\{1, \operatorname{tr}\left(C_{1}\right)-1\right\}=\left\{1, \mu_{m+1}\right\}$. Now a direct calculation produces the following:

$$
\begin{aligned}
\mu_{m+1} & =\operatorname{tr}\left(C_{1}\right)-1 \\
& =\frac{(m+1) \lambda+3 m-1}{2 m}-1 \\
& =\frac{(m+1) \lambda+m-1}{2 m} .
\end{aligned}
$$

Finally, the irreducible doubly stochastic matrix $C$ has the desired spectrum $\sigma(C)=\left\{1, \mu_{2}, \ldots, \mu_{m+1}\right\}$.

The following result is obtained by a similar argument used in Theorem 2 .

Corollary 3. Let $B$ be an irreducible row (symmetric) stochastic matrix with $m$-tuple $\Lambda=\left\{1, \mu_{2}, \ldots, \mu_{m}\right\}$ as its spectrum. Denote $\bar{\Lambda}=\left\{1, c \mu_{2}, \ldots, c \mu_{m}, \mu_{m+1}=((m+1) \lambda+m-1) / 2 m\right\}$, where $c=(2 m-1+\lambda) / 2 m$. Then $\bar{\Lambda}$ can be realized by an irreducible row (symmetric) stochastic matrix.

Notice that a real $n$-tuple $\Lambda=\left\{\lambda_{1}, \lambda_{2}, \ldots, \lambda_{n}\right\}$ is realized by an irreducible doubly stochastic matrix $A$ only if $\max \left\{\lambda_{1}, \ldots, \lambda_{n}\right\}=1=\rho(A)$ is a simple eigenvalue of $A$. For convenience, we always assume that $1=\lambda_{1}>\lambda_{2} \geq \lambda_{3} \geq$ $\cdots \geq \lambda_{n} \geq-1$.

Corollary 4. If the real triple $\Lambda=\left\{1, \lambda_{2}, \lambda_{3}\right\}\left(1>\lambda_{2} \geq \lambda_{3} \geq\right.$ -1) satisfies

$$
2+\lambda_{2}+3 \lambda_{3} \geq 0
$$

then $\Lambda$ is realized by a symmetric irreducible stochastic matrix.

Proof. Assume that $\Lambda$ satisfies Condition (13) which implies that $\lambda_{2} \geq-0.5$. Let $m=2, \lambda_{2}=\mu_{m+1}=((m+1) \lambda+m-$ $1) / 2 m=(3 \lambda+1) / 4$; then $\lambda=\left(4 \lambda_{2}-1\right) / 3 \in[-1,1), c=$ $(2 m-1+\lambda) / 2 m=(3+\lambda) / 4=\left(11+4 \lambda_{2}\right) / 12>0$. Let $c \mu_{2}=\lambda_{3}$ and

$$
B=\frac{1}{2}\left(\begin{array}{cc}
1+\lambda_{3} & 1-\lambda_{3} \\
1-\lambda_{3} & 1+\lambda_{3}
\end{array}\right)
$$

Then $B$ is an irreducible doubly stochastic matrices with $\sigma(B)=\left\{1, \lambda_{3}\right\}$ by Lemma 1 and

$$
\begin{aligned}
c B & =c\left(\begin{array}{ll}
\frac{1+\lambda_{3} / c}{2} & \frac{1-\lambda_{3} / c}{2} \\
\frac{1-\lambda_{3} / c}{2} & \frac{1+\lambda_{3} / c}{2}
\end{array}\right)=\left(\begin{array}{cc}
\frac{c+\lambda_{3}}{2} & \frac{c-\lambda_{3}}{2} \\
\frac{c-\lambda_{3}}{2} & \frac{c+\lambda_{3}}{2}
\end{array}\right) \\
& =\left(\begin{array}{ll}
\frac{8+4 \lambda_{2}+12 \lambda_{3}}{24} & \frac{8+4 \lambda_{2}-12 \lambda_{3}}{24} \\
\frac{8+4 \lambda_{2}-12 \lambda_{3}}{24} & \frac{8+4 \lambda_{2}+12 \lambda_{3}}{24}
\end{array}\right) \\
& =\left(\begin{array}{ll}
\frac{2+\lambda_{2}+3 \lambda_{3}}{6} & \frac{2+\lambda_{2}-3 \lambda_{3}}{6} \\
\frac{2+\lambda_{2}-3 \lambda_{3}}{6} & \frac{2+\lambda_{2}+3 \lambda_{3}}{6}
\end{array}\right)
\end{aligned}
$$

is nonnegative. Finally, the matrix of order $m+1=3$ in (7)

$$
\begin{aligned}
C & =\left(\begin{array}{ccc}
\frac{1+\lambda}{2} & \frac{1-\lambda}{2 \sqrt{2}} u_{2}^{T} \\
\frac{1-\lambda}{2 \sqrt{2}} u_{2} & c B
\end{array}\right) \\
& =\left(\begin{array}{ccc}
\frac{1+2 \lambda_{2}}{3} & \frac{1-\lambda_{2}}{3} & \frac{1-\lambda_{2}}{3} \\
\frac{1-\lambda_{2}}{3} & \frac{2+\lambda_{2}+3 \lambda_{3}}{6} & \frac{2+\lambda_{2}-3 \lambda_{3}}{6} \\
\frac{1-\lambda_{2}}{3} & \frac{2+\lambda_{2}-3 \lambda_{3}}{6} & \frac{2+\lambda_{2}+3 \lambda_{3}}{6}
\end{array}\right)
\end{aligned}
$$

is irreducible symmetric stochastic with $\sigma(C)=\left\{1, c \mu_{2}, \mu_{3}\right\}=$ $\Lambda$ by Theorem 2 .

Remark 5. Theorem 14 of [8] shows that Condition (13) is sufficient and necessary for a real triple $\Lambda=\left\{1, \lambda_{2}, \lambda_{3}\right\}$ to be doubly stochastic realizable.

Corollary 6. If a real feasible nonincreasing 4-tuple $\Lambda=$ $\left\{1, \lambda_{2}, \lambda_{3}, \lambda_{4}\right\}$ satisfies

$$
3+\lambda_{2}+2 \lambda_{3}+6 \lambda_{4} \geq 0
$$

then $\Lambda$ is realized by a symmetric irreducible stochastic matrix.

Proof. Assume that $\Lambda$ satisfies Condition (17). Then $0 \leq 3+$ $\lambda_{2}+2 \lambda_{3}+6 \lambda_{4} \leq 3+9 \lambda_{2}$ yields $\lambda_{2} \geq-1 / 3$. Let $m=3$, $\lambda_{2}=\mu_{m+1}=((m+1) \lambda+m-1) / 2 m=(4 \lambda+2) / 6$; then $\lambda=\left(3 \lambda_{2}-1\right) / 2 \in[-1,1), c=(2 m-1+\lambda) / 2 m=(5+\lambda) / 6=$ $\left(3+\lambda_{2}\right) / 4>0$. It is clear that $\lambda_{4} / c=4 \lambda_{4} /\left(3+\lambda_{2}\right) \geq-1$ since $4 \lambda_{4} /\left(3+\lambda_{2}\right)<-1$ would produce $3+\lambda_{2}+4 \lambda_{4}<0$, which, together with (17), yields

$$
3+\lambda_{2}+2 \lambda_{3}+6 \lambda_{4}>3+2 \lambda_{2}+4 \lambda_{4} \Longrightarrow \lambda_{3}+\lambda_{4}>0 \text {. }
$$

It follows that $3+2 \lambda_{2}+4 \lambda_{4} \geq 3\left(1+\lambda_{4}\right)+\left(\lambda_{3}+\lambda_{4}\right)>0$, which is a contradiction. So $1>4 \lambda_{2} /\left(3+\lambda_{2}\right) \geq 4 \lambda_{3} /\left(3+\lambda_{2}\right)=\lambda_{3} / c \geq$ 
$\lambda_{4} / c \geq-1$. Since $2+\lambda_{3} / c+3 \lambda_{4} / c=(1 / 2 c)\left(4 c+2 \lambda_{3}+6 \lambda_{4}\right)=$ $(1 / 2 c)\left(3+\lambda_{2}+2 \lambda_{3}+6 \lambda_{4}\right) \geq 0$ by (17), the following matrix: is irreducible doubly stochastic by whom $\left\{1, \lambda_{3} / c, \lambda_{4} / c\right\}=$ $\left\{1, \mu_{2}, \mu_{3}\right\}$ is realized by Corollary 4 and

$c B$

$$
B=\left(\begin{array}{ccc}
\frac{1+2 \lambda_{3} / c}{3} & \frac{1-\lambda_{3} / c}{3} & \frac{1-\lambda_{3} / c}{3} \\
\frac{1-\lambda_{3} / c}{3} & \frac{2+\lambda_{3} / c+3 \lambda_{4} / c}{6} & \frac{2+\lambda_{3} / c-3 \lambda_{4} / c}{6} \\
\frac{1-\lambda_{3} / c}{3} & \frac{2+\lambda_{3} / c-3 \lambda_{4} / c}{6} & \frac{2+\lambda_{3} / c+3 \lambda_{4} / c}{6}
\end{array}\right)
$$

$$
=\left(\begin{array}{ccc}
\frac{3+\lambda_{2}+8 \lambda_{3}}{12} & \frac{3+\lambda_{2}-4 \lambda_{3}}{12} & \frac{3+\lambda_{2}-4 \lambda_{3}}{12} \\
\frac{3+\lambda_{2}-4 \lambda_{3}}{12} & \frac{3+\lambda_{2}+2 \lambda_{3}+6 \lambda_{4}}{6} & \frac{3+\lambda_{2}+2 \lambda_{3}-6 \lambda_{4}}{6} \\
\frac{3+\lambda_{2}-4 \lambda_{3}}{12} & \frac{3+\lambda_{2}+2 \lambda_{3}-6 \lambda_{4}}{6} & \frac{3+\lambda_{2}+2 \lambda_{3}+6 \lambda_{4}}{6}
\end{array}\right)
$$

is nonnegative. Finally, the matrix of order $m+1=4$ in (7)

$$
\begin{aligned}
C & =\left(\begin{array}{ccc}
\frac{1+\lambda}{2} & \frac{1-\lambda}{2 \sqrt{3}} u_{3}^{T} \\
\frac{1-\lambda}{2 \sqrt{3}} u_{3} & c B
\end{array}\right) \\
& =\left(\begin{array}{cccc}
\frac{1+3 \lambda_{2}}{4} & \frac{1-\lambda_{2}}{4} & \frac{1-\lambda_{2}}{4} & \frac{1-\lambda_{2}}{4} \\
\frac{1-\lambda_{2}}{4} & \frac{3+\lambda_{2}+8 \lambda_{3}}{12} & \frac{3+\lambda_{2}-4 \lambda_{3}}{12} & \frac{3+\lambda_{2}-4 \lambda_{3}}{12} \\
\frac{1-\lambda_{2}}{4} & \frac{3+\lambda_{2}-4 \lambda_{3}}{12} & \frac{3+\lambda_{2}+2 \lambda_{3}+6 \lambda_{4}}{12} & \frac{3+\lambda_{2}+2 \lambda_{3}-6 \lambda_{4}}{12} \\
\frac{1-\lambda_{2}}{4} & \frac{3+\lambda_{2}-4 \lambda_{3}}{12} & \frac{3+\lambda_{2}+2 \lambda_{3}-6 \lambda_{4}}{12} & \frac{3+\lambda_{2}+2 \lambda_{3}+6 \lambda_{4}}{12}
\end{array}\right)
\end{aligned}
$$

is irreducible symmetric stochastic with $\sigma(C)=\left\{1, c \mu_{2}, c \mu_{3}\right.$, $\left.\mu_{4}\right\}=\Lambda$ by Theorem 2 . result.

Using this recursive method, we can prove the following

Corollary 7. Let $\Lambda=\left\{1, \lambda_{2}, \lambda_{3}, \lambda_{4}\right\}$ be S-feasible and satisfies

$$
12+3 \lambda_{2}+5 \lambda_{3}+10 \lambda_{4}+30 \lambda_{5} \geq 0
$$

$$
\left(\begin{array}{ccccc}
\frac{1+4 \lambda_{2}}{5} & \frac{1-\lambda_{2}}{5} & \frac{1-\lambda_{2}}{5} & \frac{1-\lambda_{2}}{5} & \frac{1-\lambda_{2}}{5} \\
\frac{1-\lambda_{2}}{5} & \frac{4+\lambda_{2}+15 \lambda_{3}}{20} & \frac{4+\lambda_{2}-5 \lambda_{3}}{20} & \frac{4+\lambda_{2}-5 \lambda_{3}}{20} & \frac{4+\lambda_{2}-5 \lambda_{3}}{20} \\
\frac{1-\lambda_{2}}{5} & \frac{4+\lambda_{2}-5 \lambda_{3}}{20} & \frac{12+3 \lambda_{2}+5 \lambda_{3}+40 \lambda_{4}}{60} & \frac{12+3 \lambda_{2}+5 \lambda_{3}-20 \lambda_{4}}{60} & \frac{12+3 \lambda_{2}+5 \lambda_{3}-20 \lambda_{4}}{60} \\
\frac{1-\lambda_{2}}{5} & \frac{4+\lambda_{2}-5 \lambda_{3}}{20} & \frac{12+3 \lambda_{2}+5 \lambda_{3}-20 \lambda_{4}}{60} & \frac{12+3 \lambda_{2}+5 \lambda_{3}+10 \lambda_{4}+30 \lambda_{5}}{60} & \frac{12+3 \lambda_{2}+5 \lambda_{3}+10 \lambda_{4}-30 \lambda_{5}}{60} \\
\frac{1-\lambda_{2}}{5} & \frac{4+\lambda_{2}-5 \lambda_{3}}{20} & \frac{12+3 \lambda_{2}+5 \lambda_{3}-20 \lambda_{4}}{60} & \frac{12+3 \lambda_{2}+5 \lambda_{3}+10 \lambda_{4}-30 \lambda_{5}}{60} & \frac{12+3 \lambda_{2}+5 \lambda_{3}+10 \lambda_{4}+30 \lambda_{5}}{60}
\end{array}\right),
$$

then $\Lambda$ can be realized by a symmetric irreducible stochastic matrix.

Proof. Assume that $\Lambda$ satisfies Condition (22) which implies that $\lambda_{2} \geq-1 / 4$. Let $m=4, \lambda_{2}=\mu_{m+1}=((m+1) \lambda+m-$ 1) $/ 2 m=(5 \lambda+3) / 8$; then $\lambda=\left(8 \lambda_{2}-3\right) / 5 \in[-1,1), c=(2 m-$ $1+\lambda) / 2 m=(7+\lambda) / 8=\left(4+\lambda_{2}\right) / 5>0$. Now under Condition (22) using the same recursive method and Theorem 2 , we can construct the following irreducible symmetric stochastic matrix:

$$
\text { (23) }
$$

by whom $\Lambda$ is realized. 
The next result shows that under some stronger conditions, we can construct a $3 \times 3$ nonsymmetric irreducible doubly stochastic matrix to realize the given real triple.

Proposition 8. Let $\Lambda=\left\{1, \lambda_{2}, \lambda_{3}\right\}$ satisfy $1>\lambda_{2} \geq \lambda_{3} \geq-1$. If

$$
\lambda_{2} \lambda_{3}+2 \lambda_{2}+2 \lambda_{3}+1>0
$$

then $\Lambda$ is realized by a $3 \times 3$ nonsymmetric irreducible doubly stochastic matrix.

Proof. If $\Lambda$ satisfies Condition (24), then construct the following bunch of $3 \times 3$ irreducible matrices with one parameter $p$ and trace of $1+\lambda_{2}+\lambda_{3}$ whose row sums are all equal to 1 :

$$
A(p)=\left(\begin{array}{ccc}
p & 1-p & 0 \\
1+\lambda_{2}+\lambda_{3}-3 p & p & 2 p-\lambda_{2}-\lambda_{3} \\
2 p-\lambda_{2}-\lambda_{3} & 0 & 1+\lambda_{2}+\lambda_{3}-2 p
\end{array}\right) .
$$

Then $\operatorname{det}(A(p))=-6 p^{2}+3\left(2+\lambda_{2}+\lambda_{3}\right) p-1-2\left(\lambda_{2}+\lambda_{3}\right)$ and $\operatorname{det}(A(p))=\lambda_{2} \lambda_{3}$ become $p^{2}-\left(\left(2+\lambda_{2}+\lambda_{3}\right) / 2\right) p+\left(\lambda_{2} \lambda_{3}+\right.$ $\left.2\left(\lambda_{2}+\lambda_{3}\right)+1\right) / 6=0$ which has a positive zero as follows:

$$
\begin{aligned}
p^{*}= & \frac{2+\lambda_{2}+\lambda_{3}}{4} \\
& -\sqrt{\left(\frac{2+\lambda_{2}+\lambda_{3}}{4}\right)^{2}-\frac{\lambda_{2} \lambda_{3}+2 \lambda_{2}+2 \lambda_{3}+1}{6}} \\
= & \frac{2+\lambda_{2}+\lambda_{3}}{4} \\
& -\sqrt{\frac{2\left(1-\lambda_{2}\right)^{2}+2\left(1-\lambda_{3}\right)^{2}+\left(\lambda_{2}-\lambda_{3}\right)^{2}}{48}} .
\end{aligned}
$$

Moreover,

$$
\begin{aligned}
& p^{*}<\frac{2+\lambda_{2}+\lambda_{3}}{2}<1, \\
2 p^{*}-\lambda_{2}-\lambda_{3}= & \frac{1-\lambda_{2}}{2}+\frac{1-\lambda_{3}}{2} \\
& -\sqrt{\frac{2\left(1-\lambda_{2}\right)^{2}+2\left(1-\lambda_{3}\right)^{2}+\left(\lambda_{2}-\lambda_{3}\right)^{2}}{48}} \\
\geq & \sqrt{\frac{\left(1-\lambda_{2}\right)^{2}+\left(1-\lambda_{3}\right)^{2}}{4}} \\
& -\sqrt{\frac{2\left(1-\lambda_{2}\right)^{2}+2\left(1-\lambda_{3}\right)^{2}+\left(\lambda_{2}-\lambda_{3}\right)^{2}}{48}}
\end{aligned}
$$

$$
\begin{gathered}
=\sqrt{\frac{11\left(1-\lambda_{2}\right)^{2}+11\left(1-\lambda_{3}\right)^{2}+\left(\lambda_{2}-\lambda_{3}\right)^{2}}{48}} \\
-\sqrt{\frac{2\left(1-\lambda_{2}\right)^{2}+2\left(1-\lambda_{3}\right)^{2}+\left(\lambda_{2}-\lambda_{3}\right)^{2}}{48}}
\end{gathered}
$$

$\geq 0$.

In addition, since

$$
\begin{gathered}
6\left(1-\lambda_{2}\right)^{2}+6\left(1-\lambda_{3}\right)^{2}+3\left(\lambda_{2}-\lambda_{3}\right)^{2}-\left(\lambda_{2}+\lambda_{3}-2\right)^{2} \\
=4\left(1-\lambda_{3}\right)^{2}+4\left(\lambda_{2}-\lambda_{3}\right)^{2}+4\left(\lambda_{2}-\lambda_{3}\right)^{2}>0,
\end{gathered}
$$

we have

$$
\begin{aligned}
1+ & \lambda_{2}+\lambda_{3}-3 p^{*} \\
= & \frac{\lambda_{2}+\lambda_{3}-2}{4} \\
& +\frac{1}{4} \sqrt{6\left(1-\lambda_{2}\right)^{2}+6\left(1-\lambda_{3}\right)^{2}+3\left(\lambda_{2}-\lambda_{3}\right)^{2}}
\end{aligned}
$$

$>0$.

Therefore, $A\left(p^{*}\right)$ is an irreducible doubly stochastic matrix with $\operatorname{tr}\left(A\left(p^{*}\right)\right)=1+\lambda_{2}+\lambda_{3}, \operatorname{det}\left(A\left(p^{*}\right)\right)=\lambda_{2} \lambda_{3}$ and hence $\sigma\left(A\left(p^{*}\right)\right)=\Lambda$.

Remark 9. Corollary 6 produces the sufficient condition (13) for an irreducible symmetric stochastic matrix of order 3 to have the prescribed real spectrum, and Proposition 8 produces the sufficient condition (24) for an irreducible nonsymmetric doubly stochastic matrix of order 3 to have the prescribed real spectrum. Note that Condition (24) implies Condition (13) because $2+\lambda_{2}+3 \lambda_{3}=1+2 \lambda_{2}+2 \lambda_{3}+\lambda_{2} \lambda_{3}+$ $\left(1-\lambda_{2}\right)\left(1+\lambda_{3}\right) \geq 0$ if $1+2 \lambda_{2}+2 \lambda_{3}+\lambda_{2} \lambda_{3} \geq 0$.

Theorem $\mathbf{M}$ (see [7]). Let $\Lambda^{\prime}=\left\{1, \lambda_{2}^{\prime}, \lambda_{3}^{\prime}, \lambda_{4}^{\prime}\right\}$ with $-1 \leq$ $\lambda_{2}^{\prime}, \lambda_{3}^{\prime}, \lambda_{4}^{\prime} \leq 1$. Then $\Lambda^{\prime}$ is realized by a symmetric doubly stochastic matrix with zero trace if and only if $1+\lambda_{2}^{\prime}+\lambda_{3}^{\prime}+\lambda_{4}^{\prime}=$ 0 , and when $\Lambda^{\prime}$ satisfies the condition, the matrix is

$$
B=\left(\begin{array}{cccc}
0 & 1+\lambda_{2}^{\prime} & 1+\lambda_{3}^{\prime} & 1+\lambda_{4}^{\prime} \\
1+\lambda_{2}^{\prime} & 0 & 1+\lambda_{4}^{\prime} & 1+\lambda_{3}^{\prime} \\
1+\lambda_{3}^{\prime} & 1+\lambda_{4}^{\prime} & 0 & 1+\lambda_{2}^{\prime} \\
1+\lambda_{4}^{\prime} & 1+\lambda_{3}^{\prime} & 1+\lambda_{2}^{\prime} & 0
\end{array}\right) .
$$

Corollary 10. Let $\Lambda=\left\{1, \lambda_{2}, \lambda_{3}, \lambda_{4},-1 / 4\right\},-1 \leq \lambda_{2}, \lambda_{3}, \lambda_{4} \leq$ 1 and $c=3 / 4$. If

$$
-1 \leq \frac{\lambda_{2}}{c}, \frac{\lambda_{3}}{c}, \frac{\lambda_{4}}{c} \leq 1, \quad \lambda_{2}+\lambda_{3}+\lambda_{4}=-c,
$$

then $\Lambda$ is realized by a symmetric doubly stochastic matrix with zero trace. 
Proof. Let $\lambda_{k}^{\prime}=\lambda_{k} / c, k=2,3,4$. If (32) holds, then we have $-1 \leq \lambda_{2}^{\prime}, \lambda_{3}^{\prime}, \lambda_{4}^{\prime} \leq 1$ and $1+\lambda_{2}^{\prime}+\lambda_{3}^{\prime}+\lambda_{4}^{\prime}=0$. So $\left\{1, \lambda_{2}^{\prime}, \lambda_{3}^{\prime}, \lambda_{4}^{\prime}\right\}=$ $\left\{1, \lambda_{2} / c, \lambda_{3} / c, \lambda_{4} / c\right\}$ is realized by the $4 \times 4$ doubly stochastic matrix $B$ given in (31) by Theorem $M$. Let $m=4,-1 / 4=\lambda_{5}=$ $(m+1+\lambda) / 2 m=(5+\lambda) / 8$; then $\lambda=-1, c=(2 m-1+\lambda) / 2 m=$ $3 / 4$. Now $\left\{1, c \lambda_{2}, c \lambda_{3}, c \lambda_{4}\right\}=\Lambda$ is realized by the $5 \times 5$ matrix (given in (7)) as follows:

$$
C=\left(\begin{array}{cc}
\frac{1+\lambda}{2} & \frac{1-\lambda}{4} u_{4}^{T} \\
\frac{1-\lambda}{4} u_{4} & c B
\end{array}\right)=\left(\begin{array}{cc}
0 & \frac{1}{2} u_{4}^{T} \\
\frac{1}{2} u_{4} & c B
\end{array}\right)
$$

that is an irreducible doubly stochastic with zero by Theorem 2 .

\section{The Case of Complex Spectrum}

Given a circulant doubly stochastic matrix, it is easy to obtain its spectrum (see Lemma 11). In this section, we use this result to construct an IDS (irreducible doubly stochastic) matrix to realize a given complex triple containing a pair of conjugate complex numbers with some additional conditions. This matrix is used together with Theorem 2 to construct an IDS matrix to realize a given complex 4-tuple and a 5-tuple containing exactly a pair of conjugate complex numbers with special conditions in a recursive method. Also constructed is an IDS realization of a given complex 5-tuple, which contains two pairs of conjugate complex numbers with special conditions.

The following result is well known. We give a short proof for completeness.

Lemma 11. In the complex plane, let $\Omega_{n}$ be the regular polygon whose vertices are all the nth roots of unity as follows: $q_{k}=$ $\cos (2 k \pi / n)+i \sin (2 k \pi / n), k=0,1,2, \ldots, n-1, i=\sqrt{-1}$, and let $p=u+i v(\nu \neq 0)$ be a nonreal number. If $p \in \Omega_{n}$, or equivalently, $p$ is a convex combination of the nth roots of unity; that is, $p=\lambda_{0} q_{0}+\lambda_{1} q_{1}+\cdots+\lambda_{n-1} q_{n-1}, \sum_{k=0}^{n-1} \lambda_{k}=1$, $\lambda_{k} \geq 0, k=0,1, \ldots, n-1$; then there is a doubly stochastic matrix $C_{n}$ such that

$$
\begin{aligned}
p & \in \sigma\left(C_{n}\right) \\
& =\left\{\lambda_{0}+\lambda_{1} q_{k}+\lambda_{2} q_{k}^{2}+\cdots+\lambda_{n-1} q_{k}^{n-1}, k=0,1, \ldots, n-1\right\} .
\end{aligned}
$$

Proof. It is clear that the following permutation matrix:

$$
P_{n}=\left(\begin{array}{cccccc}
0 & 1 & & & & \\
& 0 & 1 & & & \\
& & 0 & \ddots & & \\
& & & \ddots & \ddots & \\
& & & & \ddots & 1 \\
1 & & & & & 0
\end{array}\right)
$$

has spectrum $\sigma\left(P_{n}\right)=\left\{q_{0}, q_{1}, \ldots, q_{n-1}\right\}$ and then the following circulant matrix:

$$
\begin{aligned}
C_{n} & =\left(\begin{array}{cccc}
\lambda_{0} & \lambda_{1} & \cdots & \lambda_{n-1} \\
\lambda_{n-1} & \lambda_{0} & \cdots & \lambda_{n-2} \\
\vdots & \vdots & \ddots & \vdots \\
\lambda_{1} & \lambda_{2} & \cdots & \lambda_{0}
\end{array}\right) \\
& =\lambda_{0} I_{n}+\lambda_{1} P_{n}+\cdots+\lambda_{n-1} P_{n}^{n-1}
\end{aligned}
$$

is doubly stochastic and has spectrum $\sigma\left(C_{n}\right)=\left\{\lambda_{0}+\lambda_{1} q_{k}+\right.$ $\left.\lambda_{2} q_{k}^{2}+\cdots+\lambda_{n-1} q_{k}^{n-1}, k=0,1, \ldots, n-1\right\}$. When $k=1$, we have $\lambda_{0}+\lambda_{1} q_{1}+\lambda_{2} q_{1}^{2}+\cdots+\lambda_{n-1} q_{1}^{n-1}=p \in \sigma\left(C_{n}\right)$.

Theorem 12. Let $\Lambda=\left\{1, \lambda_{2}=u+i v, \lambda_{3}=u-i v\right\}$ with $u \in \mathbf{R}$ and $v>0$. Then $\Lambda$ can be realized by an IDS matrix if and only if

$$
-\frac{1}{2} \leq u<1, \quad v \leq \frac{1-u}{\sqrt{3}}
$$

When (37) holds $\Lambda$ to be realized by the irreducible doubly stochastic matrix,

$$
C_{3}=\left(\begin{array}{ccc}
\alpha & \beta & 1-\alpha-\beta \\
1-\alpha-\beta & \alpha & \beta \\
\beta & 1-\alpha-\beta & \alpha
\end{array}\right),
$$

where

$$
\alpha=\frac{1+2 u}{3}<1, \quad \beta=\frac{1-u}{3}\left(1-\frac{\sqrt{3} v}{1-u}\right) .
$$

Proof. Assume that (37) holds. Then, in the complex plane, $u+i v$ is inside the regular triangle whose vertices are all the 3 rd roots of unit $q_{0}=1, q_{1}=\cos (2 \pi / 3)+i \sin (2 \pi / 3)=-1 / 2+$ $i(\sqrt{3} / 2)$, and $q_{2}=\cos (4 \pi / 3)+i \sin (4 \pi / 3)=-1 / 2-i(\sqrt{3} / 2)$, and hence $u+i v$ is a convex combination of $q_{0}, q_{1}$, and $q_{2}$. It is not difficult to calculate

$$
u+i v=\alpha+\beta q_{1}+(1-\alpha-\beta) q_{2}
$$

where $\alpha, \beta$ is given by (39). Therefore the spectrum $\sigma\left(C_{3}\right)$ of the irreducible doubly stochastic matrix $C_{3}$ given in (38) contains $u+i v$ by Lemma 11 . Since $C_{3}$ is a doubly stochastic matrix, we have $\sigma\left(C_{3}\right)=\Lambda$ and hence the sufficiency is proved. To prove the necessity, assume that $\Lambda$ is realized by a doubly stochastic matrix $C=\left(c_{r s}\right), c_{r s} \geq 0, r, s=1, \ldots, n$. Then $1+2 u=t c C \geq 0$ from which follows $u \geq-1 / 2$, and the sum of products of pairs eigenvalues of $C$ is

$$
\begin{aligned}
2 u+u^{2}+v^{2} & =\sum_{1 \leq r<s \leq 3} \operatorname{det}\left(\begin{array}{ll}
c_{r r} & c_{r s} \\
c_{s r} & c_{s s}
\end{array}\right) \leq \sum_{1 \leq r<s \leq 3} c_{r r} c_{s s} \\
& \leq \frac{1}{3}\left(\sum_{r=1}^{3} c_{r r}\right)^{2}=\frac{1}{3}(t c C)^{2}=\frac{1}{3}(1+2 u)^{2},
\end{aligned}
$$

from which follows $3 v^{2} \leq(1-u)^{2}$. Therefore, (37) holds. 
Remark 13. The necessary and sufficient condition (37) for the $3 \times 3$ DSIEP was given by Theorems 12 and 14 of [9]. ies.

Using Theorems 2 and 12, we have the following corollar-

Corollary 14. Let $\Lambda=\left\{1, \lambda_{2}, \lambda_{3}=u+i v, \lambda_{4}=u-i v\right\}, 1>$ $\lambda_{2} \geq-1, u, v(>0) \in R$, and $c=\left(3+\lambda_{2}\right) / 4$. If

$$
-\frac{1}{2} \leq \frac{u}{c}<1, \quad \frac{v}{c} \leq \frac{1-u / c}{\sqrt{3}},
$$

then $\Lambda$ can be realized by a $4 \times 4$ irreducible stochastic matrix.

Proof. Let $m=3, \lambda_{2}=((m+1) \lambda+m-1) / 2 m=(4 \lambda+2) / 6$; then $\lambda=\left(3 \lambda_{2}-1\right) / 2 \in(-1,1), c=(2 m-1+\lambda) / 2 m=$ $(5+\lambda) / 6=\left(3+\lambda_{2}\right) / 4>0$. If $(42)$ holds, then $\{1,(u+i v) / c,(u-$ $i v) / c\}$ is realized by the irreducible doubly stochastic matrix

$$
B=\left(\begin{array}{ccc}
\alpha & \beta & 1-\alpha-\beta \\
1-\alpha-\beta & \alpha & \beta \\
\beta & 1-\alpha-\beta & \alpha
\end{array}\right)
$$

by Theorem 12, where

$$
\alpha=\frac{1+2 u / c}{3}, \quad \beta=\frac{1-u / c}{3}\left(1-\frac{\sqrt{3} v / c}{1-u / c}\right) .
$$

Now, the $4 \times 4$ matrix (given in (7)) as follows:

$$
C=\left(\begin{array}{cc}
\frac{1+\lambda}{2} & \frac{1-\lambda}{2 \sqrt{3}} u_{3}^{T} \\
\frac{1-\lambda}{2 \sqrt{3}} u_{3} & c B
\end{array}\right)
$$

is an irreducible doubly stochastic matrix $b$ whom $\{1, c((u+$ $\left.i v) / c), c((u-i v) / c), \lambda_{2}\right\}=\Lambda$ is realized (Theorem 2$)$.

Corollary 15. Let $\Lambda=\left\{1, \lambda_{2}, \lambda_{3}, u+i v, u-i v\right\}$ be a complex 5-tuple with $1>\lambda_{2} \geq \lambda_{3} \geq-1, u^{2}<u^{2}+v^{2} \leq 1, v>0$, and $c^{*}=\left(4+\lambda_{3}\right) / 5, c^{\prime}=\left(3+\lambda_{2} / c^{*}\right) / 4, c=c^{*} c^{\prime}$, then $c^{*} \in$ $(11 / 15,1), c^{\prime}>7 / 11,0<c<c^{\prime}$. If

$$
\begin{aligned}
\lambda_{3} \geq-\frac{1}{4}, \quad 1 & >\frac{\lambda_{2}}{c} \geq-1, \quad\left(\frac{u}{c}\right)^{2}+\left(\frac{v}{c}\right)^{2} \leq 1, \\
-\frac{1}{2} & \leq \frac{u}{c}, \quad \frac{v}{c} \leq \frac{1-u / c}{\sqrt{3}},
\end{aligned}
$$

then $\Lambda$ is realized by a $5 \times 5$ irreducible stochastic matrix.

Proof. Let $m=4, \lambda_{3}=((m+1) \lambda+m-1) / 2 m=(5 \lambda+3) / 8$; then $\lambda=\left(8 \lambda_{3}-3\right) / 5 \in(-1,1), c^{*}=(2 m-1+\lambda) / 2 m=(7+$ $\lambda) / 8=\left(4+\lambda_{3}\right) / 5 \in(3 / 4,1)$ and hence $c^{\prime} \geq(3-4 / 3) / 4=5 / 12$. If $\Lambda$ satisfies Condition (46), then $\left\{1, \lambda_{2} / c^{*},(u+i v) / c^{*},(u-\right.$ $\left.i v) / c^{*}\right\}$ is realized by an irreducible $4 \times 4$ doubly stochastic matrix $B^{*}$ by Corollary 14 . Now for $\lambda=\left(8 \lambda_{3}-3\right) / 5$, the $5 \times 5$ matrix (given in (7))

$$
C=\left(\begin{array}{cc}
\frac{1+\lambda}{2} & \frac{1-\lambda}{4} u_{4}^{T} \\
\frac{1-\lambda}{4} u_{4} & c^{*} B^{*}
\end{array}\right)
$$

is an irreducible doubly stochastic by whom $\left\{1, c^{*}\left(\lambda_{2} /\right.\right.$ $\left.\left.c^{*}\right), c^{*}\left((u+i v) / c^{*}\right), c^{*}\left((u-i v) / c^{*}\right), \lambda_{3}\right\}=\Lambda$ is realized by Theorem 2 .

Theorem 16. Let $\Lambda=\left\{\lambda_{1}, \lambda_{2}, \lambda_{3}, \lambda_{4}\right\}=\left\{1,-2 u-1, u+i v, \lambda_{3}=\right.$ $u-i v\}, u, v(>0) \in R$ contain a pair of conjugate complex numbers such that $\lambda_{1}+\lambda_{2}+\lambda_{3}+\lambda_{4}=0$. If

$$
-\frac{1}{2} \leq u \leq 0, \quad 1+u-v \geq 0
$$

then $\Lambda$ is realized by a $4 \times 4$ irreducible doubly stochastic matrix with zero trace.

Proof. Assume that (48) holds. Then, in the complex plane, $u+i v$ is inside the right triangle whose vertices are $q_{1}=i$, $q_{2}=-1, q_{3}=-i$ and hence $u+i v$ is a convex combination of $q_{1}, q_{2}$, and $q_{3}$. It is not difficult to calculate

$$
u+i v=\frac{1+u-v}{2} q_{1}+(-u) q_{2}+\frac{1+u+v}{2} q_{3}
$$

Since $q_{0}=1, q_{1}=i, q_{2}=-1, q_{3}=-i$ are all the 4 th roots of unit, Lemma 11 asserts that the spectrum of the following $4 \times 4$ irreducible doubly stochastic matrix:

$$
\left(\begin{array}{cccc}
0 & \frac{1+u-v}{2} & -u & \frac{1+u+v}{2} \\
\frac{1+u+v}{2} & 0 & \frac{1+u-v}{2} & -u \\
-u & \frac{1+u+v}{2} & 0 & \frac{1+u-v}{2} \\
\frac{1+u-v}{2} & -u & \frac{1+u+v}{2} & 0
\end{array}\right)
$$

is $\left\{((1+u-v) / 2) i^{k}-u(-1)^{k}+((1+u+v) / 2)(-i)^{k}, k=0,1,2,3\right\}=$ $\{1, u-i v,-1-2 u, u+i v\}=\Lambda$.

Corollary 17. Let $\Lambda=\left\{\lambda_{1}, \lambda_{2}, \lambda_{3}, \lambda_{4}, \lambda_{5}\right\}=\{1,-1 / 4,(-3-$ $8 u) / 4, u+i v, u-i v\}, u, v(>0) \in R$ and $c=\left(3+\lambda_{2}\right) / 4=11 / 16$. If

$$
-\frac{1}{2} \leq \frac{u}{c} \leq 0, \quad 1+\frac{u}{c}-\frac{v}{c} \geq 0,
$$

then $\Lambda$ is a realized by a $5 \times 5$ irreducible stochastic matrix.

Proof. Let $m=4, \lambda_{2}=((m+1) \lambda+m-1) / 2 m=(5 \lambda+3) / 8$; then $\lambda=-1$. If (51) holds, then $\{1,-1-2 u / c,(u+i v) / c,(u-$ $i v) / c\}$ is realized by the irreducible doubly stochastic matrix 


$$
B=\left(\begin{array}{cccc}
0 & \frac{1+u / c-v / c}{2} & -u / c & \frac{1+u / c+v / c}{2} \\
\frac{1+u / c+v / c}{2} & 0 & \frac{1+u / c-v / c}{2} & -u / c \\
-u / c & \frac{1+u / c+v / c}{2} & 0 & \frac{1+u / c-v / c}{2} \\
\frac{1+u / c-v / c}{2} & -u / c & \frac{1+u / c+v / c}{2} & 0
\end{array}\right)
$$

by Theorem 16 . Now the following $5 \times 5$ matrix (given in $(7)$ ):

$$
C=\left(\begin{array}{cc}
\frac{1+\lambda}{2} & \frac{1-\lambda}{4} u_{4}^{T} \\
\frac{1-\lambda}{4} u_{4} & c B
\end{array}\right)=\left(\begin{array}{cc}
0 & \frac{1}{2} u_{4}^{T} \\
\frac{1}{2} u_{4} & c B
\end{array}\right)
$$

is an irreducible doubly stochastic matrix with zero trace by whom $\left\{1, c((u+i v) / c), c((u-i v) / c), \lambda_{2},-1-\lambda_{2}-2 u\right\}=\Lambda$ is realized by Theorem 2 .

Theorem 18. Let $\Lambda=\left\{1, \lambda_{2}=u+i v, \lambda_{3}=u-i v, \lambda_{4}=\right.$ $\left.u^{\prime}+i v^{\prime}, \lambda_{5}=u-i v^{\prime}\right\}$ contain two pairs of conjugate complex numbers with $u, v(>0)$ given and $u^{\prime}, v^{\prime}(>0)$ depending on $u, v$ $(i=\sqrt{-1})$. If

$$
\begin{gathered}
\cos \frac{4 \pi}{5} \leq u<1, \\
v<\min \left\{\frac{\sin (2 \pi / 5)(u-1)}{\cos (2 \pi / 5)-1}, \cos (4 \pi / 5)\right. \\
\left.+\frac{(\sin (2 \pi / 5)-\sin (4 \pi / 5))(u-\cos (2 \pi / 5))}{\cos (2 \pi / 5)-\cos (4 \pi / 5)}\right\},
\end{gathered}
$$

then $\Lambda$ is realized by a $5 \times 5$ irreducible doubly stochastic matrix, where $u^{\prime}, v^{\prime}$ are depending on $u, v$ (see (58) and (63)).

Proof. Assume that (54) holds. Then, in the complex plane, $u+i v$ is inside the right pentagon whose vertices are all the 5 th roots of units $q_{0}=1, q_{k}=\cos (2 k \pi / 5)+i \sin (2 k \pi / 5)$, $k=1,2,3,4$ (see Figure 1 ) and hence $u+i v$ is a convex combination of $q_{0}, q_{1}, q_{2}, q_{3}, q_{4}$. There are two cases to be considered.

Case 1. $u$, $v$ satisfy $(54)$ and $v \leq \sin (4 \pi / 5)(u-1) /(\cos (4 \pi / 5)-$ 1). In this case, $u+i v$ is inside Triangle $\Delta q_{0} q_{2} q_{3}$ and hence $u+i v$ is a convex combination of $q_{0}, q_{2}, q_{3}$. A calculation yields

$$
u+i v=\lambda_{0}+\lambda_{2} q_{2}+\lambda_{3} q_{3}
$$

where

$$
\begin{gathered}
\lambda_{0}=\frac{u-\cos (4 \pi / 5)}{1-\cos (4 \pi / 5)} \\
\lambda_{2}=\frac{1-u}{1-\cos (4 \pi / 5)}\left(\frac{1}{2}-\frac{(1-\cos (4 \pi / 5)) v}{2 \sin (4 \pi / 5)(1-u)}\right) \\
\lambda_{3}=1-\lambda_{0}-\lambda_{2} .
\end{gathered}
$$

Now the following irreducible circulant matrix that is also doubly stochastic:

$$
C_{5}=\left(\begin{array}{ccccc}
\lambda_{0} & 0 & \lambda_{2} & \lambda_{3} & 0 \\
0 & \lambda_{0} & 0 & \lambda_{2} & \lambda_{3} \\
\lambda_{3} & 0 & \lambda_{0} & 0 & \lambda_{2} \\
\lambda_{2} & \lambda_{3} & 0 & \lambda_{0} & 0 \\
0 & \lambda_{2} & \lambda_{3} & 0 & \lambda_{0}
\end{array}\right)
$$

has spectrum: $\sigma\left(C_{5}\right)=\left\{\lambda_{0}+\lambda_{2} q_{k}^{2}+\lambda_{3} q_{k}^{3}, k=0,1,2,3,4\right\}$ by Lemma 11. Taking $k=0,1,4$, we have $1, u \pm i v \in \sigma\left(C_{3}\right)$ and taking $k=2,3$, we have that $\lambda_{0}+\lambda_{2} q_{4}+\lambda_{3} q_{1}$ and $\lambda_{0}+\lambda_{2} q_{1}+$ $\lambda_{3} q_{4}$ are in $\sigma\left(C_{3}\right)$ and are conjugate to each other. Therefore, if we set

$$
\begin{gathered}
u^{\prime}=\lambda_{0}+\lambda_{2} \cos \frac{8 \pi}{5}+\lambda_{3} \cos \frac{2 \pi}{5}, \\
v^{\prime}=\left|\lambda_{2} \sin \frac{8 \pi}{5}+\lambda_{3} \sin \frac{2 \pi}{5}\right|,
\end{gathered}
$$

then $\Lambda$ is realized by the $5 \times 5$ irreducible doubly stochastic matrix $C_{5}$.

Case 2. $u, v$ satisfy $(54)$ and $v>\sin (4 \pi / 5)(u-1) /(\cos (4 \pi / 5)-$ 1). In this case, $u+i v$ is inside Triangle $\Delta q_{0} q_{1} q_{2}$ and hence $u+i v$ is a convex combination of $q_{0}, q_{1}, q_{2}$. A calculation yields

$$
u+i v=\lambda_{0}^{\prime}+\lambda_{1}^{\prime} q_{1}+\lambda_{2}^{\prime} q_{2}
$$

where

$$
\begin{gathered}
\lambda_{0}^{\prime}=\frac{u-x}{1-x}, \\
\lambda_{1}^{\prime}=\frac{(x-\cos (4 \pi / 5))\left(1-\lambda_{0}^{\prime}\right)}{\cos (2 \pi / 5)-\cos (4 \pi / 5)}, \\
\lambda_{2}^{\prime}=1-\lambda_{0}^{\prime}-\lambda_{1}^{\prime},
\end{gathered}
$$

with

$$
\begin{aligned}
x= & \left(\left(\sin \frac{2 \pi}{5}-\left(\frac{\sin (2 \pi / 5)-\sin (4 \pi / 5)}{\cos (2 \pi / 5)-\cos (4 \pi / 5)}\right)\right.\right. \\
& \left.\left.\times \cos \frac{2 \pi}{5}\right)(u-1)+v\right) \\
& \times\left(\left(\frac{\sin (2 \pi / 5)-\sin (4 \pi / 5)}{\cos (2 \pi / 5)-\cos (4 \pi / 5)}\right)(1-u)+v\right)^{-1} .
\end{aligned}
$$




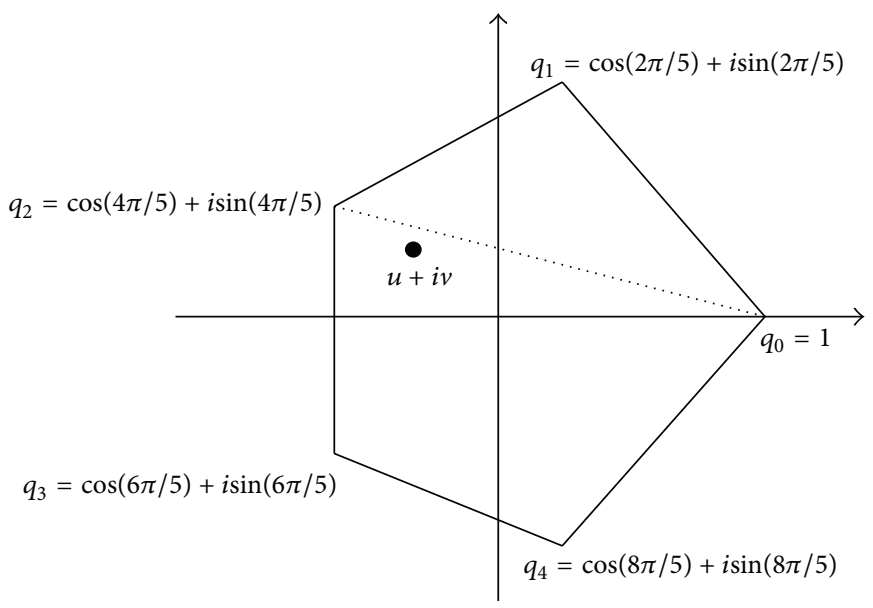

Figure 1

Now the irreducible circulant matrix that is also doubly stochastic as follows:

$$
C_{5}^{\prime}=\left(\begin{array}{ccccc}
\lambda_{0}^{\prime} & \lambda_{1}^{\prime} & \lambda_{2}^{\prime} & 0 & 0 \\
0 & \lambda_{0}^{\prime} & \lambda_{1}^{\prime} & \lambda_{2}^{\prime} & 0 \\
0 & 0 & \lambda_{0}^{\prime} & \lambda_{1}^{\prime} & \lambda_{2}^{\prime} \\
\lambda_{2}^{\prime} & 0 & 0 & \lambda_{0}^{\prime} & \lambda_{1}^{\prime} \\
\lambda_{1}^{\prime} & \lambda_{2}^{\prime} & 0 & 0 & \lambda_{0}^{\prime}
\end{array}\right)
$$

has spectrum $\sigma\left(C_{5}^{\prime}\right)=\left\{\lambda_{0}^{\prime}+\lambda_{1}^{\prime} q_{k}+\lambda_{2}^{\prime} q_{k}^{2}, k=0,1,2,3,4\right\}$ by Lemma 11. Taking $k=0,1,4$, we have $1, u \pm i v \in \sigma\left(C_{3}^{\prime}\right)$ and taking $k=2,3$, we have that $\lambda_{0}^{\prime}+\lambda_{1}^{\prime} q_{2}+\lambda_{2}^{\prime} q_{4}$ and $\lambda_{0}^{\prime}+\lambda_{1}^{\prime} q_{3}+$ $\lambda_{2}^{\prime} q_{1}$ are in $\sigma\left(C_{3}^{\prime}\right)$ and are conjugate to each other. Therefore, if we set

$$
\begin{gathered}
u^{\prime}=\lambda_{0}^{\prime}+\lambda_{1}^{\prime} \cos \frac{4 \pi}{5}+\lambda_{2}^{\prime} \cos \frac{8 \pi}{5}, \\
v^{\prime}=\left|\lambda_{1}^{\prime} \sin \frac{4 \pi}{5}+\lambda_{2}^{\prime} \sin \frac{8 \pi}{5}\right|,
\end{gathered}
$$

then $\Lambda$ is realized by the $5 \times 5$ irreducible doubly stochastic matrix $C_{5}^{\prime}$.

Example 19. $\Lambda_{1}=\{1,-0.3+0.6 i,-0.3-0.6 i\}$ satisfies Condition (37) of Theorem 12 and is doubly stochastic realized by

$$
A_{1}=\left(\begin{array}{lll}
0.13333 & 0.08692 & 0.77974 \\
0.77974 & 0.13333 & 0.08692 \\
0.08692 & 0.77974 & 0.13333
\end{array}\right)
$$

Example 20. Let $\Lambda_{2}=\left\{1, \lambda_{2}, u+v i, u-v i\right\}=\{1,0.46,-0.3+$ $0.6 i,-0.3-0.6 i\}, c=\left(3+\lambda_{2}\right) / 4=0.865$. Then Condition (42) of Corollary 14 is satisfied and $\Lambda_{2}$ is doubly stochastic realized by

$$
A_{2}=\left(\begin{array}{cccc}
0.595 & 0.135 & 0.135 & 0.135 \\
0.135 & 0.08833 & 0.04192 & 0.73744 \\
0.135 & 0.73744 & 0.08833 & 0.04192 \\
0.135 & 0.04192 & 0.73744 & 0.08833
\end{array}\right)
$$

Example 21. Let $\Lambda_{3}=\left\{1, \lambda_{2}, \lambda_{3}, u+v i, u-v i\right\}=$ $\{1,0.6,0.1,-0.3+0.6 i,-0.3-0.6 i\}$. Then $c^{*}=\left(4+\lambda_{3}\right) / 5=$ $0.82, c^{\prime}=\left(3+\lambda_{2} / c^{*}\right) / 4=0.93295, c=c^{*} c^{\prime}=0.765$, and Condition (46) of Corollary 15 is satisfied and $\Lambda_{3}$ is doubly stochastic realized by

$$
A_{3}=\left(\begin{array}{ccccc}
0.28 & 0.18 & 0.18 & 0.18 & 0.18 \\
0.18 & 0.655 & 0.055 & 0.055 & 0.055 \\
0.18 & 0.055 & 0.055 & 0.00859 & 0.70141 \\
0.18 & 0.055 & 0.70141 & 0.055 & 0.00859 \\
0.18 & 0.055 & 0.00859 & 0.70141 & 0.055
\end{array}\right) .
$$

Example 22. Let $\Lambda_{4}=\{1,-1 / 4,(-3-8 u) / 3, u+i v, u-i v\}$ with $u=-0.2, v=0.4$ and $c=3 / 4$. Then Condition (51) of Corollary 17 is satisfied and $\Lambda_{4}$ is doubly stochastic realized by

$$
A_{4}=\left(\begin{array}{ccccc}
0 & 0.25 & 0.25 & 0.25 & 0.25 \\
0.25 & 0 & 0.475 & 0.2 & 0.075 \\
0.25 & 0.075 & 0 & 0.475 & 0.2 \\
0.25 & 0.2 & 0.075 & 0 & 0.475 \\
0.25 & 0.475 & 0.2 & 0.075 & 0
\end{array}\right)
$$

Example 23. Let $\Lambda_{5}=\left\{1, u+v i, u-v i, u^{\prime}+v^{\prime} i, u^{\prime}-\right.$ $\left.v^{\prime} i\right\}$, where $u=0.3, v=0.6$ and $u^{\prime}, v^{\prime}(>0)$ will be determined later. It is easy to verify that $u, v$ satisfy Condition (54) of Theorem 18 and $v>\sin (4 \pi / 5)(u-$ 1) $/(\cos (4 \pi / 5)-1)$ (i.e., $\Lambda_{4}$ belongs to Case 2) and $u+$ $v i=\lambda_{0}+\lambda_{1} q_{1}+\lambda_{2} q_{2}=0.29613+0.51278(\cos (2 \pi / 5)+$ $\sin (2 \pi / 5) i)+0.19109(\cos (4 \pi / 5)+\sin (4 \pi / 5) i)$. Let $u^{\prime}=\lambda_{0}+$ $\lambda_{1} \cos (2 \pi / 5)+\lambda_{2} \cos (4 \pi / 5)=-0.05966, v^{\prime}=\mid \lambda_{1} \sin (2 \pi / 5)+$ $\lambda_{2} \sin (4 \pi / 5) \mid=0.11967$. Then $\Lambda_{4}=\left\{1, u+v i, u-v i, u^{\prime}+\right.$ $\left.v^{\prime} i, u^{\prime}-v^{\prime} i\right\}$ is doubly stochastic realized by the following irreducible doubly stochastic matrix:

$$
A_{5}=\left(\begin{array}{ccccc}
0.29613 & 0.51278 & 0.19109 & 0 & 0 \\
0 & 0.29613 & 0.51278 & 0.19109 & 0 \\
0 & 0 & 0.29613 & 0.51278 & 0.19109 \\
0.19109 & 0 & 0 & 0.29613 & 0.51278 \\
0.51278 & 0.19109 & 0 & 0 & 0.29613
\end{array}\right) .
$$




\section{Conflict of Interests}

The authors declare that there is no conflict of interests regarding the publication of this paper.

\section{Acknowledgments}

The authors thank very much the anonymous referee whose valuable comments and suggestions helped them to improve the representation of the paper. The first author's work is supported by The Doctorate Point Foundation of the Educational Ministry of China (no. 20113401130001) and the National Natural Science Foundation of China-Guangdong Joint Found (no. U1201255). Changqing Xu is supported by the China State Natural Science Foundation Monumental Project (no. 6119010) and the China National Natural Science Foundation Key Project (no. 61190114)

\section{References}

[1] A. Borobia, "On the nonnegative eigenvalue problem," Linear Algebra and Its Applications, vol. 223/224, pp. 131-140, 1995.

[2] M. Fang, "A note on the inverse eigenvalue problem for symmetric doubly stochastic matrices," Linear Algebra and Its Applications, vol. 432, no. 11, pp. 2925-2927, 2010.

[3] S.-G. Hwang and S.-S. Pyo, "The inverse eigenvalue problem for symmetric doubly stochastic matrices," Linear Algebra and Its Applications, vol. 379, pp. 77-83, 2004.

[4] I. Kaddoura and B. Mourad, "On a conjecture concerning the inverse eigenvalue problem of $4 \times 4$ symmetric doubly stochastic matrices," International Mathematical Forum, vol. 31, no. 3, pp. 1513-1519, 2008.

[5] T. J. Laffey and E. Meehan, "A characterization of trace zero nonnegative $5 \times 5$ matrices," Linear Algebra and Its Applications, vol. 302/303, pp. 295-302, 1999.

[6] R. Loewy and D. London, "A note on an inverse problem for nonnegative matrices," Linear and Multilinear Algebra, vol. 6, no. 1, pp. 83-90, 1978.

[7] B. Mourad, "On a spectral property of doubly stochastic matrices and its application to their inverse eigenvalue problem," Linear Algebra and Its Applications, vol. 436, no. 9, pp. 34003412, 2012.

[8] A. M. Nazari and F. Sherafat, "On the inverse eigenvalue problem for nonnegative matrices of order two to five," Linear Algebra and Its Applications, vol. 436, no. 7, pp. 1771-1790, 2012.

[9] H. Perfect and L. Mirsky, "Spectral properties of doublystochastic matrices," Monatshefte für Mathematik, vol. 69, pp. 35-57, 1965.

[10] R. Reams, "An inequality for nonnegative matrices and the inverse eigenvalue problem," Linear and Multilinear Algebra, vol. 41, no. 4, pp. 367-375, 1996.

[11] O. Rojo and R. L. Soto, "Existence and construction of nonnegative matrices with complex spectrum," Linear Algebra and Its Applications, vol. 368, pp. 53-69, 2003.

[12] J. Torre-Mayo, M. R. Abril-Raymundo, E. Alarcia-Estévez, C. Marijuán, and M. Pisonero, "The nonnegative inverse eigenvalue problem from the coefficients of the characteristic polynomial. EBL digraphs," Linear Algebra and Its Applications, vol. 426, no. 2-3, pp. 729-773, 2007.
[13] S. Yang and X. Li, "Inverse eigenvalue problems of $4 \times 4$ irreducible nonnegative matrices," in Advances in Matrix Theory and Applications. Proceedings of the 8th International Conference on Matrix theory and Applications, vol. I, World Academic Union, 2008.

[14] S. Yang and C. Xu, "Row stochastic inverse eigenvalue problem," Journal of Inequalities and Applications, vol. 2011, article 24, 5 pages, 2011.

[15] R. A. Horn and C. R. Johnson, Matrix Analysis, Cambridge University Press, Cambridge, UK, 1985. 


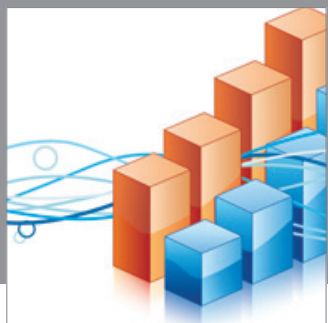

Advances in

Operations Research

mansans

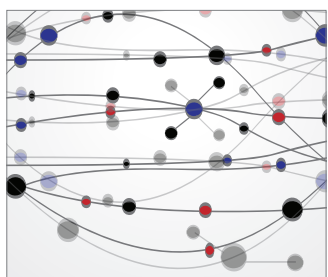

The Scientific World Journal
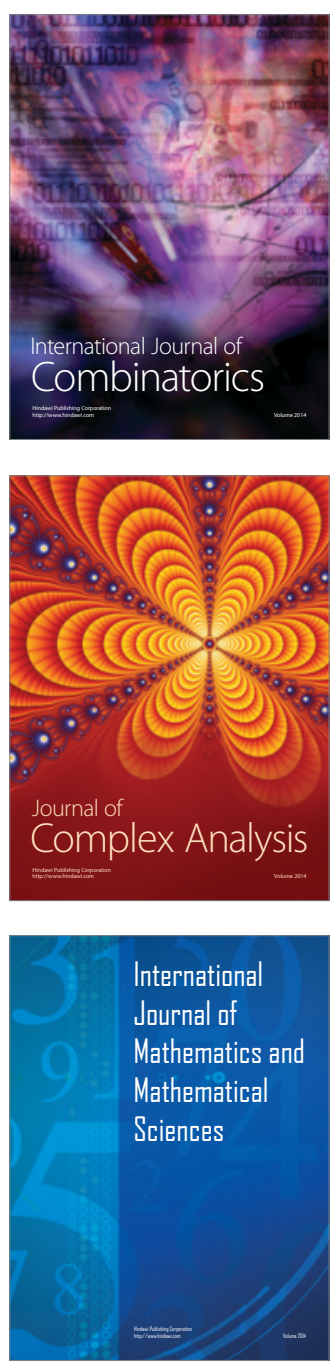
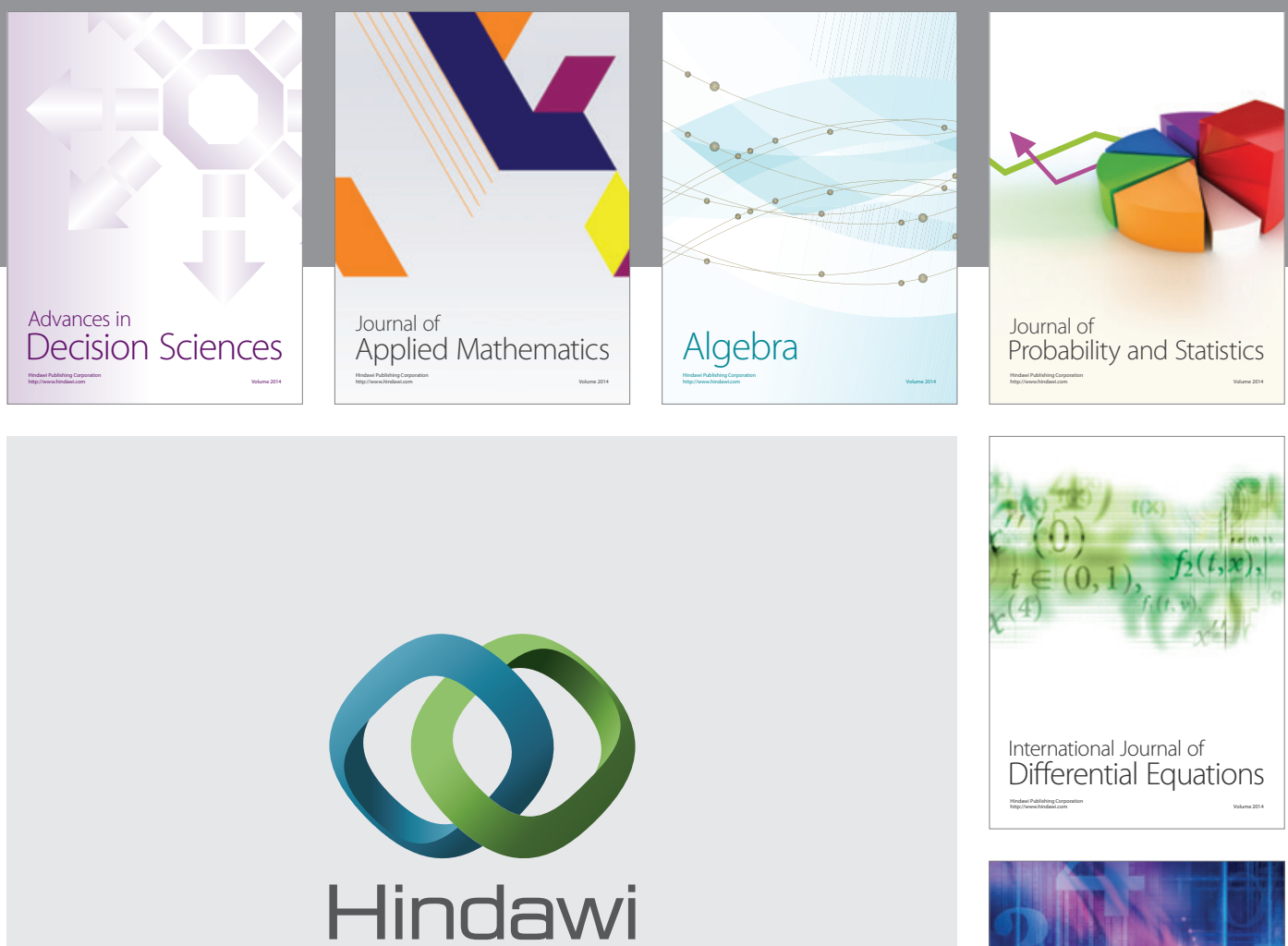

Submit your manuscripts at http://www.hindawi.com
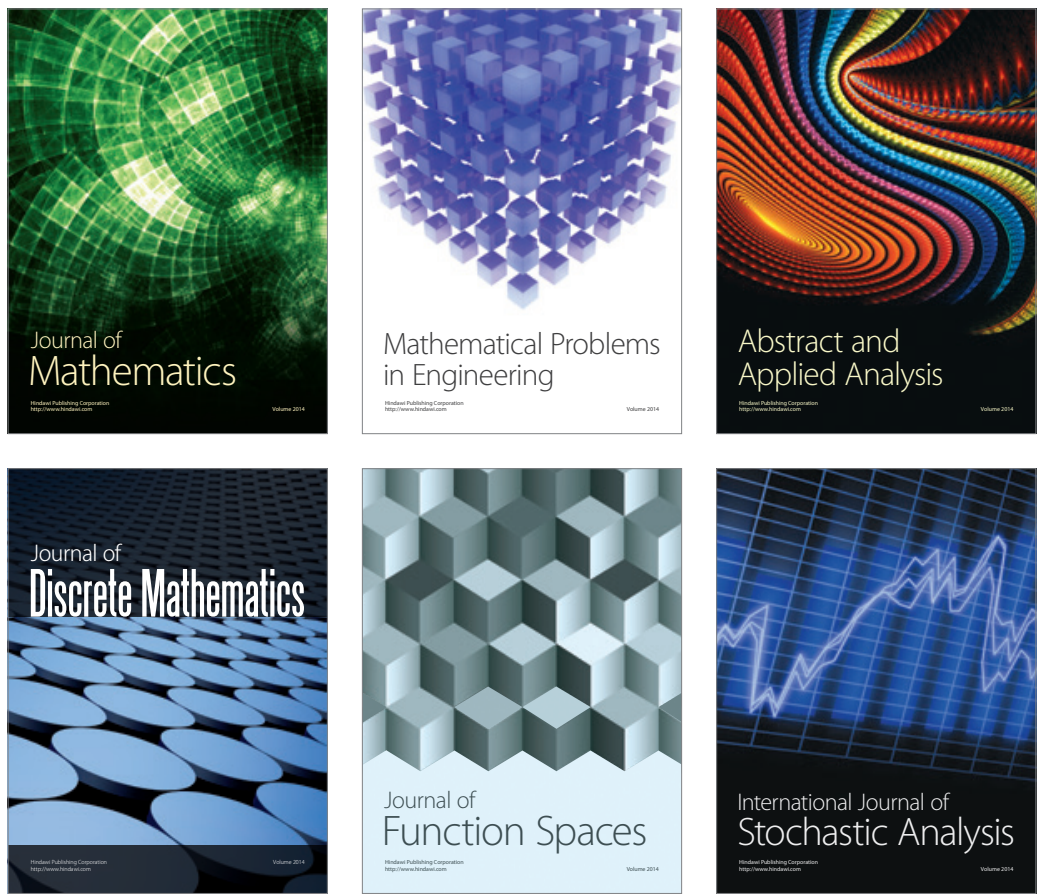

Journal of

Function Spaces

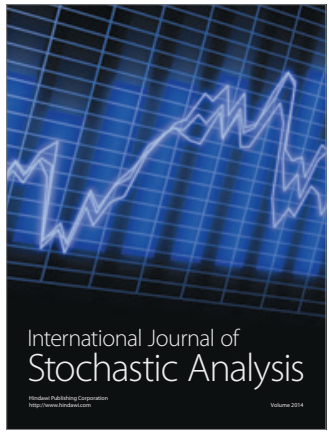

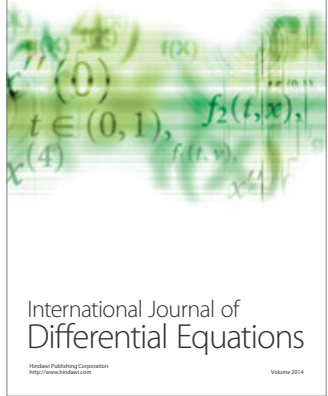
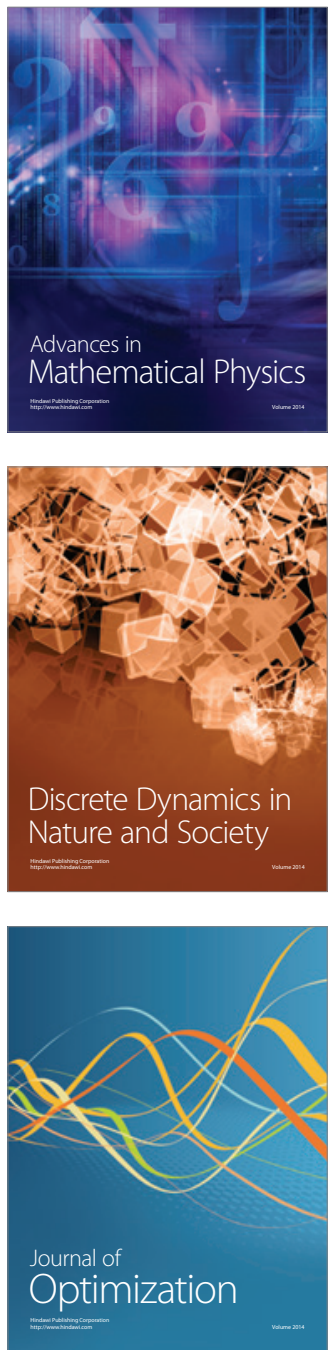